\title{
Identification of differentially expressed genes of primary spermatocyte against round spermatid isolated from human testis using the laser capture microdissection technique
}

\author{
Gang LIANG ${ }^{1,4}$, Xiao Dong ZHANG ${ }^{1}$, Lu Jing WANG ${ }^{1}$, Yu Shen SHA ${ }^{2}$, Jian Chao ZHANG ${ }^{2}$, Shi Ying MIAO ${ }^{1}$, \\ Shu Dong ZONG ${ }^{2}$, Lin Fang WANG ${ }^{1, *}$, S.S. KOIDE ${ }^{3}$ \\ ${ }^{I}$ National Laboratory Medical Molecular Biology, Institute of Basic Medical Sciences, Chinese Academy of Medical Sciences, \\ Peking Union Medical College, 5 Dong Dan San Tiao, 100005 Beijing, China \\ ${ }^{2}$ National Research Institute for Family Planning, WHO Collaboration Center for Research in Human Reproduction, Beijing, 12 Da Hui \\ Si, 100081 Beijing, China \\ ${ }^{3}$ Center for Biomedical Research, Population Council, 1230 York Avenue, New York, NY 10021, USA \\ ${ }^{4}$ Chinese National Human Genome Center, Beijing, 3-707 North Yong Chang Road BDA, Beijing 100176, China
}

\begin{abstract}
The method of laser capture microdissection (LCM) combined with suppressive subtractive hybridization (SSH) was developed to isolate specific germ cells from human testis sections and to identify the genes expressed during differentiation and development. In the present study, over 10,000 primary spermatocytes and round spermatid cells were successfully isolated by LCM. Using the cDNAs from primary spermatocytes and round spermatids, SSH cDNAs library of primary spermatocyte-specific was constructed. The average insert size of the cDNA isolated from 75 randomly picked white clones was $500 \mathrm{bp}$, ranging from $250 \mathrm{bp}$ to $1.7 \mathrm{~kb}$. Using the dot-blot method, a total of 421 clones were examined, resulting in the identification of 390 positive clones emitting strong signals. Partial sequence of cDNAs prepared from each clone was determined with an overall success rate of $84.4 \%$. Genes encoding cytochrome c oxidase II and the rescue factor-humanin were most frequently expressed in primary spermatocytes, suggesting their roles involved in meiosis.
\end{abstract}

Keywords: laser capture microdissection, suppressive subtractive hybridization, spermatogenesis, cytochrome c oxidase II, humanin.

\section{INTRODUCTION}

Spermatogenesis is a complex orderly process, whereby diploid stem cells undergo differentiation and meiosis to be transformed into mature haploid spermatozoa [1]. Differentiation of germ cells occurs while situated within the seminiferous tubules of human testis. The development of one generation of spermatogonia, spermatocytes, and spermatids is closely integrated with other generations presented in the same region of the tubule. Spermatogenesis is acknowledged to be a process encompassing both time and space [2]; whereby genes are expressed under stringent temporal and spatial

\footnotetext{
*Correspondence: Lin Fang WANG,

Tel: +86-10-65296418, Fax: +86-10-65240529

E-mail: wanglf@ms.imicams.ac.cn
}

regulations. Thus identifying the differentially expressed genes will be of immense value in delineating the regulatory mechanism of spermatogenesis. A prerequisite to pursue a study of gene expression in different cell types which continue to vary with time and in spatial arrangement as with spermatogenesis is to perfect a method of isolating specific germ cells in sufficient quantity and purity from the testis, a complex, heterogenous tissue.

The technology of laser capture microdissection (LCM) brought about a revolution in the methods of cell separation $[3,4,5]$. It is a new technique originated at the NIH, and commercially developed through a Collaborative Research and Development Agreement partnership with Arcturus Engineering Inc. It's a reliable, rapid method of isolating specific and pure population of cells or even a single cell from a complex tissue. The principle of LCM entails the application of a transparent ethylene vinyl 
acetate (EVA) thermoplastic film to the surface of the tissue section. Under the microscope, the tissue is viewed through the film and the clusters of cells to be captured are selected. When the appropriate cells are centered in the field of view, the operator pushes a button that activates a laser diode integral with the microscope optics. The pulsed laser beam activates a precise spot on the EVA film immediately above the selected cells. At this precise location, the film melts and fuses with the underlying cells of choice. When the film is removed, the selected cell(s) adhere to its undersurface, while the surrounding tissue remains unattached. This technique has been utilized in the Cancer Genome Anatomy Project of the USA $[6,7]$.

LCM provides an opportunity to isolate germ cells in specific stages of differention, allowing identification of specific expressed genes. Primary spermatocyte is a diploid cell which undergoes meiosis to produce two secondary spermatocytes, while round spermatid is a haploid cell derived from a secondary spermatocyte undergoing meiosis II, which subsequently differentiate into elongated spermatozoa. On obtaining pure primary spematocytes and round spermatids, the gene cluster or specific genes involved with meiosis can be determined. Also, by identifying the genes expressed in these cells, factors involved in the regulation of morphological structural changes to spermatozoa can be elucidated.

In the present study, pure populations of primary spermatocytes and round spermatids were captured from sections of human testis using LCM. Libraries of primary spermatocyte-specific/enriched cDNAs were constructed, utilizing the technique of suppression subtractive hybridization (SSH). A total of 390 positive DNAs were sequenced. Comparing the differentially expressed genes of primary spermatocyte against round spermatid, factors associated with the regulation of meiosis and spermatogenesis were identified.

\section{MATERIALS AND METHODS}

\section{Tissue samples and LCM}

Testis tissue used in this study was obtained from the Beijing Hospital (Beijing, China) after obtaining a signed informing consent and the approval of the project by the Institutional Review Board. The tissue was immediately embedded in optimal cutting compound (OCT) medium after surgery and subsequently in liquid nitrogen. Using a cryostat, tissue sections were cut to $8 \mu \mathrm{m}$ thickness and placed on RNAse-free microscope slides. Tissues were stained with Mayer's reagent. The pure population of primary spermatocytes (pachytene) from Stage IV or V and round spermatids from Stage I or II of human spermatogenesis cycle [8] were microdissected using the LCM (LM200, Arcturus Engineering, Mountain View, CA, USA) system according to the manufacturer's instructions (Fig. 1). Approximately a set of 10,000 cells were microdissected.
First-strand cDNA synthesis and cDNA amplification by long distance (LD) PCR

Total RNAs were extracted from the LCM-captured cells using Trizol $^{\circledR}$ Reagent (GIBCO BRL). The RNAs from primary spermatocyte and round spermatid cells were used as templates to reverse transcribe the first-strand cDNA using the SMART ${ }^{\mathrm{TM}}$ PCR cDNA Synthesis Kit (Clontech, Palo Alto, CA, USA) according to the manufacturer's protocol. Then the cDNA was amplified by LD PCR. Briefly, a sample of $2 \mu 1$ of the first-strand cDNA mixture was transferred to a tube containing $100 \mu \mathrm{l}$ of PCR reaction system: $80 \mu \mathrm{l}$ deonized water, $10 \mu 1$ 10×advantage 2 PCR buffer, $2 \mu 150 \times$ dNTP mix, $4 \mu 1$ PCR primer (5'-AAGCAGTGGTATCAACGCAG AGT$\left.3^{\prime}\right)$ and $2 \mu 150 \times$ advantage 2 polymerase mix. The PCR products were separated by electrophoresis on a $1.1 \%$ agarose/EtBr gel in $1 \times$ TAE buffer and further purified by chromatography on a CHROMA SPIN+TE-400 Column (Clontech, Palo Alto, CA, USA).

\section{Preparation of tester and driver cDNA}

Tester ds cDNAs were prepared from the above PCR products of primary spermatocytes, using CLONTECH PCR-Select ${ }^{\mathrm{TM}}$ cDNA Subtraction Kit (Clontech, Palo Alto, CA, USA). The cDNAs were first digested with $R s a$ I to yield short blunt-ended fragments, which were ligated with an adaptor at the ends, according to the Manufacturer' protocol. Driver ds cDNAs were prepared from round spermatids and blunt-ended by digesting with RsaI. The ends were kept intact.

\section{Subtractive hybridization}

To obtain differentially expressed cDNAs, two rounds of hybridizations were performed. The purpose of the first round hybridization was to equalize and to enrich the differentially expressed sequences. The objective of the second round was to produce double-stranded tester molecules with different adaptors on each end.

\section{Suppression of PCR amplification}

Two rounds of PCR amplifications were performed. In the first amplification, PCR was suppressed; whereby only differentially expressed sequences can be amplified exponentially. In the second procedure, the background was reduced to enrich the differentially expressed sequences. Each PCR product was analyzed on a $2.0 \%$ agarose/EtBr gel.

\section{Cloning of subtracted cDNA}

The subtracted cDNAs obtained from the second PCR amplification were cloned into pGEM $^{\circledR}$-T Easy vector (Promega, Madison, WI, USA) and transformed into E. coli DH5 $\alpha$ cells. The transformed cells were plated onto LB agar culture plates containing ampicillin, X-gal and IPTG. Thus, a SSH cDNA library of putative primary spermatocyte-specific/enriched was constructed. To determinate the size of the cDNA inserts, 75 clones were randomly picked and amplified with M13f and M13r primers. Each reaction product was analyzed by electrophoresis on a $1.2 \%$ agar/EtBr gel.

\section{Differential screening of subtracted cDNA library}

Preparation of $c D N A$ dot blots of the PCR products

White colonies were picked out and each clone was left to grow in $100 \mu \mathrm{lof} \mathrm{LB}^{\mathrm{Amp}+}$ medium in a $96-w e l l$ plate at $37^{\circ} \mathrm{C}$ for $3 \mathrm{~h}$ with shaking. For PCR amplification, $1 \mu \mathrm{l}$ of each bacterial culture was transferred to another 96-well plate and nested PCR was performed 
using the GeneAmp PCR System 2700 (Applied Biosystems, Foster City, CA, USA). Then $5 \mu$ of each PCR product was mixed with $5 \mu \mathrm{l}$ of $0.6 \mathrm{~N} \mathrm{NaOH}$ and $1 \mu \mathrm{l}$ of each mixture was dot-blotted onto Hybond- $\mathrm{N}^{+}$nylon membranes (Amersham Phamrmacia Biotech). The blots were neutralized with $0.5 \mathrm{M}$ Tris- $\mathrm{HCl}(\mathrm{pH} 7.5)$ for $2 \mathrm{~min}$ and washed with ddH2O. The cDNAs were cross-linked to the membrane using a GS Gene Linker ${ }^{\mathrm{TM}}$ UV Chamber (BIO-RAD, USA).

\section{Preparation of probes}

cDNA probes were prepared from tester and driver cDNAs using the Prime-a-Gene ${ }^{\circledast}$ Labeling System (Promega, Madison, WI, USA) according to the manufacturer's protocol $\left(10^{7} \mathrm{cpm}\right.$ per $\left.100 \mathrm{ng}\right)$.

\section{Hybridization}

The labeled cDNA probes were hybridized to the DNA dot-blot membranes in ExpressHyb (Clontech, Palo Alto, CA, USA) at $72{ }^{\circ} \mathrm{C}$ overnight. The membranes were washed with low-stringency $(2 \times \mathrm{SSC} / 0.5 \% \mathrm{SDS})$ solution $(4 \times 20 \mathrm{~min})$ at $68^{\circ} \mathrm{C}$ followed with high-stringency $(0.2 \times \mathrm{SSC} / 0.5 \% \mathrm{SDS})$ solution $(2 \times 20 \mathrm{~min})$ at $68^{\circ} \mathrm{C}$. The membranes were briefly air-dried and exposed to $\mathrm{x}$-ray film (Kodak, USA) at $-70^{\circ} \mathrm{C}$.

\section{DNA sequencing and homology search}

All the positive clones were sequenced with SP6/T7 primer using ABI 3700 (Applied Biosystems, Foster City, CA, USA) performed by the Automated Sequencing Facility, Chinese National Human Genome Center, Beijing. Search for nucleic acid homology was performed against the GenBank database using the advanced BLAST program [9] at the National Center for Biotechnology Information (NIH, Bethesda, MD) website (www.ncbi.nlm.nih.gov).

\section{RESULTS}

\section{Capture of primary spermatocyte and round spermatid}

A captured primary spermatocyte and a round spermatid from human testis by LCM are depicted in Fig. 1. The genes expressed in these cells were determined.

\section{Quality assessment of amplified ds cDNA from total RNA}

Using total RNA prepared from primary spermatocytes and round spermatids, ds cDNAs were synthesized using the SMART ${ }^{\mathrm{TM}}$ PCR cDNA Synthesis Kit. The quality of PCR product was assessed by electrophoresis on a $1.1 \%$ agarose gel. A homogeneous smear of each PCR product ranging from 250 to $2000 \mathrm{bp}$ in size was obtained (Fig. 2), showing the complex nature of the ds cDNAs which were used in the construction of the SSH cDNA library.

\section{Amplification of the subtracted cDNAs by PCR}

Products of the first PCR amplification were separated by electrophoresis (Fig. 3). The unsubtracted cDNAs and the subtracted cDNAs (control) provided by the kit displayed as smears (Fig. 3, lanes 2, 3); whereas, no visible smears or bands were detected with the subtracted product (Fig. 3, lane 1). On the other hand, after the second PCR

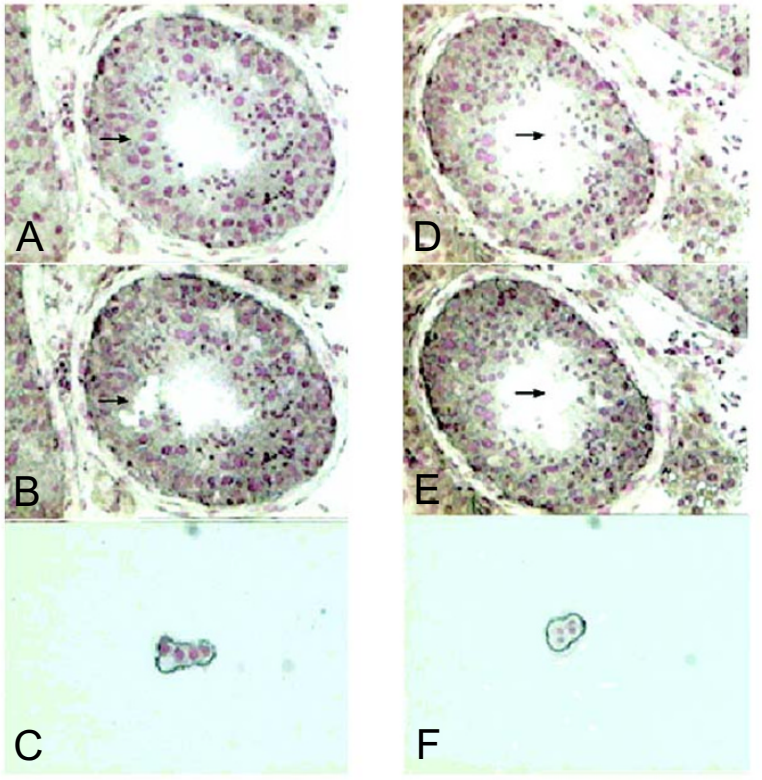

Fig. 1 Germ cells isolated by laser capture microdissection from human testis sections. A cryostat section of $8 \mu \mathrm{m}$ thickness was used. (A-C) represents primary spermatocytes and (D-F) are round spermatids. (A, D) Testis before LCM; (B, E) Testis after LCM; (C, F) Isolated captured cells.

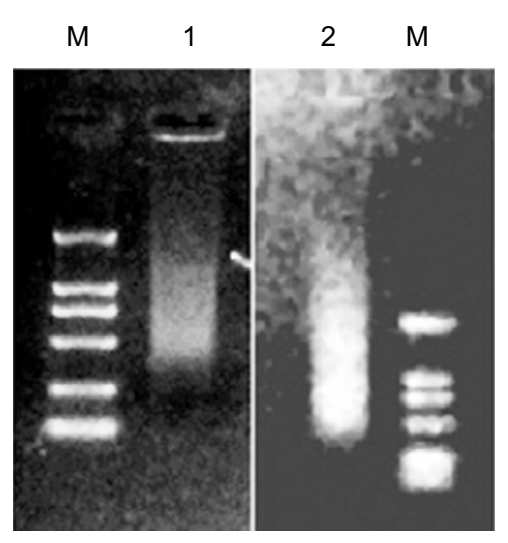

Fig. 2 ds cDNAs synthesized by LD PCR. Total RNA prepared from LCM isolated cells was used as templates. PCR product were subjected to electrophoresis on a $1.1 \%$ agarose/ $\mathrm{EtBr}$ gel. Both of the two lanes of M were DL-2000 markers, from top to bottom: 2000, $1000,750,500,250,100 \mathrm{bp}$. Lane 1, cDNA of primary spermatocytes; lane 2, cDNA of round spermatids.

amplification, the smear of the subtracted cDNAs was darker than that of the unsubtracted products, which suggests fewer cDNA types (Fig. 3, lanes 4, 5). The PCR product of the subtracted cDNAs occurred as a smear consisting of a series of fine bands ranging from 0.5 to $1 \mathrm{~kb}$ (Fig. 3, lane 4). 


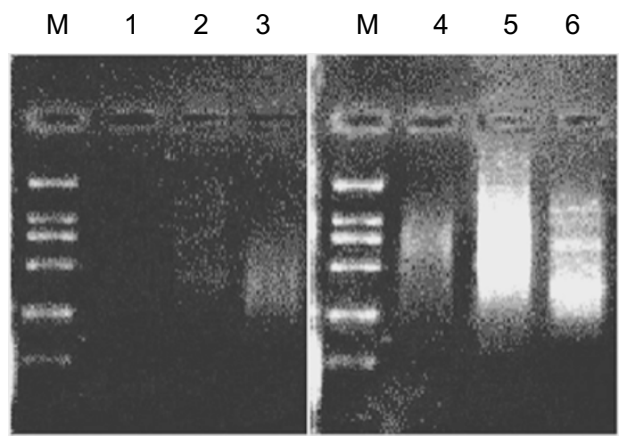

Fig. 3 PCR products of suppression subtracted cDNA. M: DL2000 markers; 1ane 1, first PCR amplified products of subtracted cDNA; lane 2, first amplified PCR products of unsubtracted control; lane 3, first amplified PCR products of subtracted cDNA control (provided by the kit); lane 4, second amplified PCR products of subtracted cDNA; lane 5, second amplified PCR products of unsubtracted control; lane 6 , second amplified PCR products of subtracted cDNA control.

\section{Cloning and identification of differential expressed cDNA}

Differential screening was performed to identify the primary spermatocyte-specific cDNAs against those of round spermatid. Using dot-blot, a total of 421 clones were spotted onto two identical nylon membranes and hybridized with tester cDNA probe from primary spermatocytes and driver cDNA from round spermatids. An example of differential dot blot screening is depicted in Fig. 4, showing 390 positive clones obtained from primary spermatocytes. Strong signals were emitted when probed with tester cDNAs (Fig. 4A), compared to driver cDNA (Fig. 4B).

\section{Search for homology in the cDNA sequences}

A total of 390 positive clones were sequenced using the SP6/T7 primer, producing 329 ESTs of satisfactory quality with an overall success rate of $84.4 \%$. The entire ESTs were subjected to a search for sequence homology in the GenBank DNA and EST database by BLAST. According to the searching results, the clones were divided into three groups of ESTs as proposed by Dey et al [10]. Group I consisted of ESTs corresponding to known genes, defined as those EST sequences sharing at least 95\% homology over at least $100 \mathrm{bp}$ of DNA sequence with known genes. Group II consisted of ESTs possessing identity (sharing at least $95 \%$ homology over at least 100 bp) with sequences in the dbEST database. Group III consisted of those ESTs having no corresponding or homologous sequence in the dbEST databases. Thus, this group of ESTs can be tentatively considered as novel cDNAs. The distribution of the EST clusters for high homology genes, high homology ESTs and novel ESTs were $58.7 \%, 1.8 \%$ and $39.5 \%$, respectively, for the primary spermatocyte-specific cDNAs. High homology genes including those that are either identical or nearly identical to previously reported genes made up to $58.7 \%$ of the human primary spermatocyte-specific subtracted cDNA library. Those high homology genes that were detected more than once are listed in Tab. 1.

\section{DISCUSSION}

New technologies have been sufficiently developed to demonstrate that changes in gene expression associated with different stages of seminiferous epithelium cycle. The transillumination method perfected by Parvinen et al allowed researchers to selectively concentrate enough pieces of tubules at different stages of the cycle [11]. This method aims to isolate different tubules at stage VIII, but not to specifically separate the spermatogonia, preleptotene spermatocytes, pachytene spermatocytes, round spermatids, elongated spermatids and Sertoli cells at that stage, as well as to investigate the respective DNAs, RNAs, and protein composition of these distinct cell populations [12]. To this day, the isolation and characterization of the germ cells and somatic cells from the testis still remains as a formidable obstacle.

LCM is an ideal method to segregate cells or groups of cells from complex tissues, which makes it acutely needed for the study of spermatogenesis. Previously, the method of LCM had been applied to study the acquisition of stagespecific tubule areas from rat testis tissue section [13]. In the present study, LCM was carried out to analyze differential gene expression in different types of human testis germ cells. Large quantities of pure populations of primary spermatocytes and round spermatids were obtained by 
Tab. 1 Clones showing high homology to known genes detected more than once

\begin{tabular}{lcc}
\hline \multicolumn{1}{c}{ Gene } & Species & Frequency \\
\hline Cytochrome c oxidase II & $\mathrm{H}$ & 7 \\
Homo sapiens humanin (HN1) & $\mathrm{H}$ & 5 \\
Similar to NADH dehydrogenase I & $\mathrm{H}$ & 3 \\
Homo sapiens Spast gene for spastin protein & $\mathrm{H}$ & 2 \\
Homo sapiens signal sequence receptor, beta & $\mathrm{H}$ & 2 \\
(Translocon-associated protein beta) (SSR2) & & \\
Heterogeneous nuclear ribonucleoprotein H3 (2H9) (HNRPH3) & $\mathrm{H}$ & 2 \\
Homo sapiens sterol carrier protein 2 (SCP2) & $\mathrm{H}$ & 2 \\
Fucosidase, alpha-L-1, tissue (FUCA1) & $\mathrm{H}$ & 2 \\
Homo sapiens golgin-67 (KIAA0855) & $\mathrm{H}$ & 2 \\
Macaca fascicularis brain cDNA, clone: QnpA-14574 & $\mathrm{M}$ & 2 \\
Homo sapiens cathelicidin antimicrobial peptide (CAMP) & $\mathrm{H}$ & 2 \\
Homo sapiens cDNA FLJ12049 fis, clone HEMBB1001996 & $\mathrm{H}$ & 2 \\
\hline
\end{tabular}

Species: H, Homo sapiens; M, Macaca fascicularis.

LCM, allowing satisfactory recovery of sufficient RNA to prepare appropriate amounts of cDNAs to be amplified by using the SMART PCR cDNA Synthesis Kit. Because of the small quantity of RNA found in LCM captured cells, increasing its yield is a prerequisite in attaining the goal of the study. Previous investigators used the modified Stratagene Microisolation protocol from NIH to construct the cDNA library [14]. In the present study, the general reagent Trizol was used; whereby the amount of RNA extracted from isolated spermatocytes and spermatids was sufficient and the quality of the mRNA was good. Moreover, since the RNA possesses an integrated 5 ' end, it can be amplified using the SMART ${ }^{\mathrm{TM}}$ kit system.

SSH is a PCR-based technique that selectively suppresses the amplification of undesirable sequences [15]. It has been extensively used to identify differentially expressed genes on a wide scale $[16,17,18]$. The normalization step equalizes the abundance of cDNAs within the target population and the subtraction step excludes the common sequences between the target and driver populations, and then allows differentially expressed transcripts to be isolated. Using the cDNAs prepared from mRNAs of primary spermatocytes and round spermatid cells, the construction of the first human SSH cDNA library of primary spermatocytespecific/enriched has been accomplished. Previously, constructed cDNA library was not cell type-specific, while LCM is relatively rapid, efficient, and precise [19, 20]. However, it is not possible to discern whether the captured cell was intact within the section, as well as to microscopically determine the level to which adjacent Setoil cell cytoplasm may be contaminated by the captured germ cells
[12]. The SSH method could exclude the common sequences (like the transcripts of Sertoil cells) between the target (primary spermatocytes) and driver populations (roud spermatids). So, the application of SSH avoids the problem of contamination with extraneous mRNAs derived from other germ cells and Sertoli cells mRNAs. The constructed cDNA subtractive library of human primary spermatocytespecific/enriched lays a solid foundation for screening and cloning new and specific genes of spermatogenesis.

By applying the method of PCR-selected differential screening, 390 positive clones of primary spermatocytespecificity have been identified and sequenced, resulting in the identification of a number of known cDNAs and novel cDNAs. Among these cDNAs, two noteworthy genes have been identified, namely, encoding cytochrome c oxidase II (COX II) and the rescue factor-humanin (HN1). They were detected in several clones signifying strong up-regulation. The COX II mRNA has been reported to be strongly expressed in pachytene spermatocytes of the testes of adult and prepubertal rats. The highest level of expression occurs in germ cells at stages VII-VIII of the spermatogenic cycle [21] and reflects a differential expression between spermatocyte and spermatid [22]. The functional significance of the high expression of COX II mRNA in pachytene spermatocytes and its stage-dependent changes is not clearly showed. This occurrence may reflect the energy demanded by these cells as they enter the final stages of meiosis. HNI is another frequently encountered gene and is also up-regulated in human primary spermatocytes. It encodes a short polypeptide and is capable of abolishing neuronal cell death caused by multiple types genes in- 
volved in Alzheimer's disease and by the $A \beta$ amyloid [23]. HN1 may be a crucial factor in preventing germ cell death and/or apoptosis thereby allowing spermatogenesis to proceed.

\section{ACKNOWLEDGMENTS}

This study was supported by grants from National Natural Science Foundation of China (No.30070173 and No. 30240019), National High Technology Research and Development Plan of China (No.2001AA221131), Special Fund for Major State Basic Research Project (No. G1999055901) and State Ministry of Science and Technology Program (2002BA711A01).

Received, Jun 30, 2003

Revised, Aug 4, 2004

Accpeted, Sep 16, 2004

\section{REFERENCES}

1 Clermont Y, Oko R, Hermo L. Cell Biology of mammalian spermatogenesis. In Desjardins C. Ed. Cell and Molecular Biology of the Testis, Oxford Univ. Press, New York 1993.

2 Kerr JB. Macro, micro, and molecular research on spermatogenesis: the quest to understand its control. Microsc Res Tech 1995; 32:364-84.

3 Simone NL, Bonner RF, Gillespie JW, Emmert-Buck MR, Liotta LA. Laser-capture microdissection: opening the microscopic frontier to molecular analysis. Trends Genet 1998; 7:272-6.

4 Bonner RF, Emmert-Buck M, Cole K, et al. Laser capture microdissection: molecular analysis of tissue. Science 1997; 278: 1481-3.

5 Emmert-Buck MR, Bonner RF, Smith PD, et al. Laser capture microdissection. Science 1997; 274:998-1001.

6 Krizman DB, Wagner L, Lash A, Strausberg RL, Emmert-Buck MR. The Cancer Genome Anatomy Project: EST sequencing and the genetics of cancer progression. Neoplasia 1999; 1:101-6.

7 Shillitoe EJ, May M, Patel V, et al. Genome-wide analysis of oral cancer-early results from the Cancer Genome Anatomy Project. Oral Oncol 2000; 36:8-16.

8 Clermont $Y$. The cycle of the seminiferouse epithelium of man. Am J Anat 1963; 112:35.

9 Altschul, Stephen F, Thomas L Madden, et al. Gapped BLAST and PSI-BLAST: a new generation of protein database search programs. Nucleic Acids Res 1997; 25: 3389-402.

10 Dey R, Son HH, Cho MI. Isolation and partial sequencing of potentially odontoblast-specific/enriched rat cDNA clones obtained by suppression subtractive hybridization. Arch Oral Biol 2001; 46:249-60.

11 Parvilien M, Ruokonen A. Endogenous steroids in the rat seminiferous tubules: comparison of the stages of the epithelial cycle isolated by transillumination-assisted microdissection. J Androl 1982; 3:211-20.

12 Suarez-Quian CA, Goldstein SR, Bonner RF. Laser capture microdissection: a new tool for the study of spermatogenesis. $\mathrm{J}$ Androl 2000; 21:601-8.

13 Sluka P, O'Donnell L, Stanton PG. Stage-Specific Expression of Genes Associated With Rat Spermatogenesis: Characterization by Laser-Capture Microdissection and Real-Time Polymerase Chain Reaction. Biol Reprod 2002; 67:820-8.

14 Krizman DB, Chuaqui RF, Meltzer PS, et al. Construction of a Representative cDNA library from Prostatic Intraepithelial Neoplasia. Cacer Res 1996; 56:5380-3.

15 Diatchenko L, Lau YF, Campbell AP, et al. Suppression subtractive hybridization: A method for generating differentially regulated or tissue-specific cDNA probes and libraries. Proc Natl Acad Sci USA 1996; 93:6025-30.

16 Liang P, Pardee AB. Differential display. A general protocol. Mol Biotechnol 1998; 10:261-7.

17 Fan LC, Yang ST, Gui JF. Differential screening and characterization analysis of the egg envelope glycoprotein ZP3 cDNAs between gynogenetic and gonochoristic crucian carp. Cell Res 2001; 11:17-27.

18 Li B, Zhang YL. Identification of up-regulated genes in human uterine leiomyoma by suppression subtractive hybridization. Cell Res 2002; 12:215-21.

19 Luo L, Saluiiga RC, Guo H, et al. Gene expression profiles of laser-captured adjacent neuronal subtypes. Nai Med 1999; 5: 117-22.

20 Jin L, Thompson CA, Qian X, et al. Analysis of anterior pituitary hormone mRNA expression in imniunophenotypically characterized single cells after laser capture microdissection. Lab Invest 1999; 79:511-2.

21 Saunders PT, Millar MR, West AP, Sharpe RM. Mitochondrial cytochrome c oxidase II messenger ribonucleic acid is expressed in pachytene spermatocytes at high levels and in a stage-dependent manner during spermatogenesis in the rat. Biol Reprod 1993; 48:57-67.

22 Savaris RF. Assessment of the degree of contamination of rat germ cell preparations using specific cDNA probes. Braz J Med Biol Res 1997; 30:347-58.

23 Hashimoto Y, Niikura T, Tajima H, et al. A rescue factor abolishing neuronal cell death by a wide spectrum of familial Alzheimer's disease genes and A $\beta$ ?. Proc Natl Acad Sci 2001; 98:6336-41. 